\title{
Simulation numérique de la dynamique du phytoplancton dans une succession de réservoirs à faible temps de rétention sur la rivière Lot $^{1}$
}

\author{
A. Dauta ${ }^{2}$ \\ J. Capblancq ${ }^{2}$
}

Mots clés : Dynamique du phytoplanton, modèle numérique, rivière.

In modèle de simulation numérique est proposé pour prédire en fonction du débit, de l'ensoleillement et des apports de nutriments, l'évolution de la biomasse de phytoplancton dans la série de plans d'eau qui se succèdent sur le cours canalisé de la rivière Lot. Ce modèle, basé sur les connaissances actuelles de la physiologie des algues, décrit en particulier les variations des constituants cellulaires (C, N, P, Chlorophylle) en fonction des conditions nutritives. La validité et la sensibilité du modèle sont testées par comparaison avec les résultats de deux séries de mesures réalisées sur une même masse d'eau, pendant son parcours vers l'aval, la première sur une distance de $50 \mathrm{~km}$, la seconde sur une distance de $200 \mathrm{~km}$.

Numerical simulation of phytoplankton dynamics in a succession of water bodies with short retention time on the river Lot.

Kevwords: Phytoplankton dynamics, numerical model, regulated river-

Changes in phytoplankton biomass in a succession of water bodies of the transformed course of the river lot are simulated by a numerical model which takes into account the influences of discharge, solar energy and nutrient inputs. This model, in accordance with present knowledge of the physiology of planktonic algae, particularly describes changes in cellular composition (C, N, P and chlorophyll content) associated with nutrient availability. Calibration and validity of the model were tested by the comparison of simulation results with field data measured by following the same mass of water on its course downstream, first during a passage of $50 \mathrm{~km}$ and then during a passage of $200 \mathrm{~km}$.

\section{Introduction}

Le cours du Lot a été progressivement modifié par l'homme d'abord pour rendre la rivière navigable puis pour produire l'électricité. Actuellement, 62 barrages de navigation - dont 25 aménagés pour la production hydroélectrique - transforment le cours moyen et inférieur en une suite de plans d'eau longs de 1 à $8 \mathrm{~km}$, profonds de 1 à 10 mètres (fig. 1). La gestion hydraulique des retenues implantées à l'amont influence les débits de ce secteur canalisé. Ces aménagements provoquent en particulier un fort ralentissement du courant en été qui, joint aux reiets d'origine domestique, industrielle ou agricole,

I. Travail réalisé dans le cadre de l'ASP a eau * du PIREN CNRS.

7. Laboratoire d'Hydrobiologie, UA 695 CNRS, Université Paul Sahatier, 118 route de Narbonne, 31062 Toulouse Cedex - France. favorise la prolifération d'algues planctoniques (Dauta 1975, Capblancq \& Dauta 1978, Décamps \& al 1984). Le principal problème posé étant de discerner le rôle respectif de ces facteurs sur la qualité de l'eau, les modèles de simulation numérique nous ont paru constituer l'outil le plus approprié à l'étude du comportement de la rivière face à ces perturbations.

Un modèle de simulation numérique a été progressivement développé à partir des premières tentatives de modélisation (Casanova-Batut 1977 ; Caussade \& al 1978 ; Delclaux 1980) et des études expérimentales qu'elles ont suscité (Guerri \& al 1981. Brunel \& al 1982, Dauta \& al 1982). Ce modèle a été testé dans un premier temps sur des séries de valeurs mesurées à l'entrée et à la sortie d'un bief du Lot (Capblancq \& al 1982). Sa transposition à une 


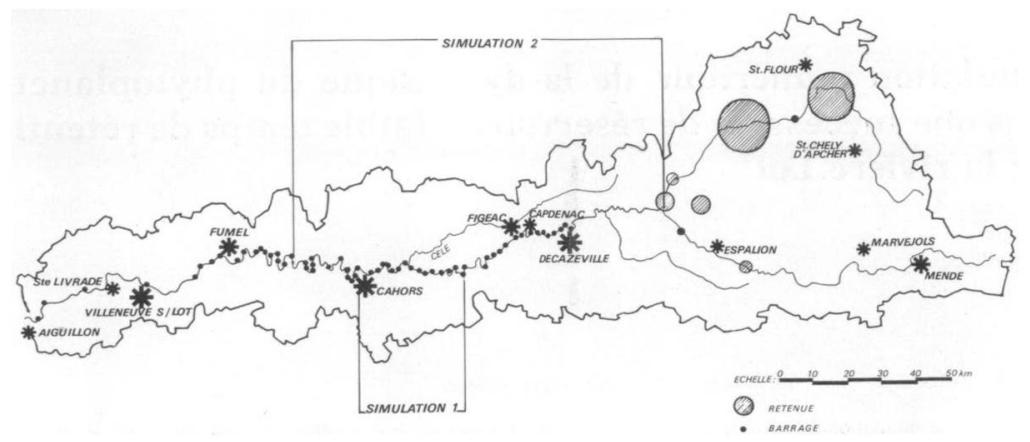

Fig. 1 : Rivière Lot : position des barrages, principaux foyers de pollution et localisation des secteurs modélisés

succession de biefs et à l'ensemble du cours canalisé fait l'objet du présent travail.

\section{Le modèle}

Le modèle élaboré se propose de simuler l'évolution de la biomasse de phytoplancton entre l'amont et l'aval du secteur canalisé en fonction du débit. des conditions climatiques et des quantités d'éléments nutritifs disponibles. La structure de ce modèle, représentée de manière très schématique dans l'organigramme de la figure 2 , peut se résumer ainsi.

En liaison avec un modèle simple d'écoulement, qui calcule l'avancement de la masse d'eau en fonction de la morphomètrie des biefs et des débits, le modèle biologique décrit la dynamique du phyto. plancton en dissociant l'absorption des éléments nutritifs minéraux ( $C, N, P)$ et l'accroissement de la binmasse (B). Le couplage entre ces deux processus est assuré par une fonction de stockage, le contenu des algues en carbone $\left(Q_{C}\right)$ en azote $\left(Q_{N}\right)$ et en phosphore $\left(Q_{p}\right)$ pouvant varier entre un minimum et un maximum qui correspondent respectivement à une situation de carence (croissance nulle) et à un apport nutritif excédentaire ("luxury uptake "). Les taux d'absorption des éléments nutritifs $\left(V_{N}, V_{P}\right)$ et de division cellulaire $(\mu)$ dépendent à chaque instant des valeurs de $Q_{C}, Q_{N}$ et $Q_{P}$ en même temps qu'ils les déterminent. Conformément aux observations faites sur des cultures (Healey \& Hendzel 1979, Rhee
1978, Dauta 1982) la teneur des cellules en Chlorophylle (QCHL) dépend également de $\mathrm{O}_{\mathrm{N}}$ et de $\mathrm{Q}_{\mathrm{P}}$ et agit sur le taux d'assimilation du carbone $\left(V_{C}\right)$. L'observation des cultures indique enfin qu'une carence prolongée en $\mathbf{N}$ ou en $\mathbf{P}$ tend à favoriser la sédimentation des algues. On aboutit à la formulation résumée dans le tableau I.

On peut reconnaître dans les équations (1) à (6) du tableau I les formulations générales utilisées par de nombreux auteurs (Lehman \& al 1975, Nyholm 1977 a et b p. ex). Par rapport au modèle proposé antérieurement par Capblancq et al (1982) quelques modifications ont été introduites à la suite de divers essais de calibration. Ainsi :

(1) La réduction du taux de multiplication $(\mu)$ est supposée suivre la " loi du minimum ", i.e. le taux de croissance est limité par le constituant cellulaire ( $\mathrm{N}$ ou $\mathrm{P}$ ) le moins abondant. Cette formulation, conforme aux résultats expérimentaux de Rhee (1978) est moins contraignante que la formule multiplicative.

(2) Les différentes expressions de f (I'o) ne donnent pas de résultats significativement différents. Cependant, il est apparu nécessaire d'inclure l'effet de la densité du phytoplancton sur le coefficient d'atténuation de la lumière dans l'eau (E). Nos mesures indiquent un coefficient d'atténuation spécifique voisin de $0,015 \mathrm{~m}^{-1} \mathrm{mg} \mathrm{CHI}^{-1} \mathrm{~m}^{-2}$.

(3) Les teneurs des algues en chlorophylle sont liées à leur degré de carence en azote $\left(Q_{N}\right)$ et, à un 


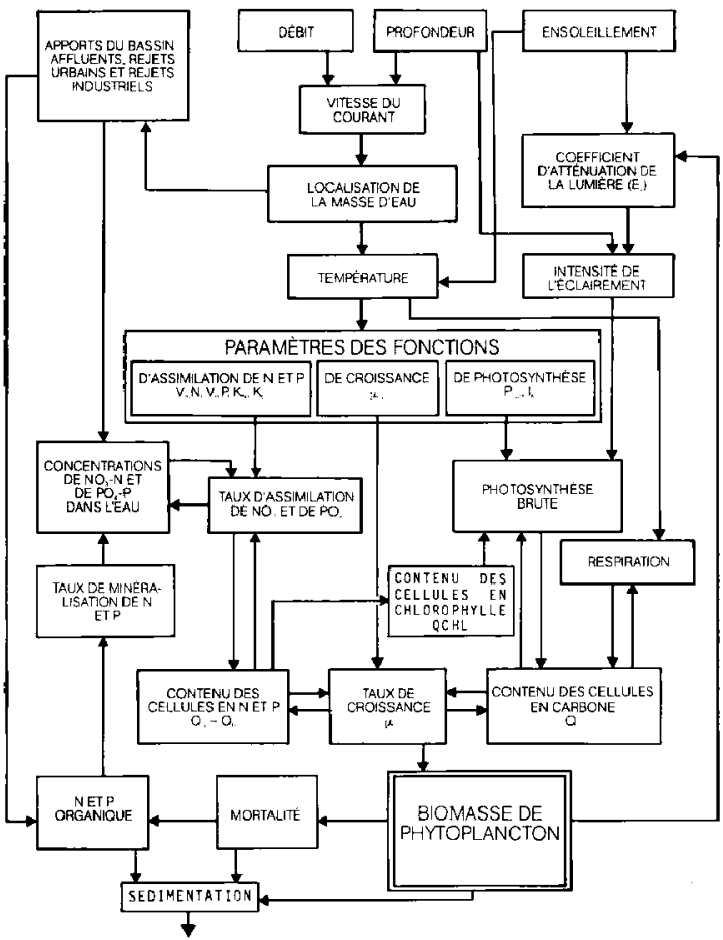

Fig. 2 : Organigramme schematisum la structure du modèle de production du phytoplancton en rivière

degré moindre, en phosphore $\left(\mathrm{Q}_{\mathrm{p}}\right)$ par une loi hyperbolique (Dauta, 1983).

(4) L'état nutritionnel agit également sur le taux de sédimentation (SED) : on sait en effet qu'une carence en $\mathrm{N}$ ou en $\mathrm{P}$ entraine l'accumulation d'hydrates de carbone et, par suite, une augmentation du volume des cellules et de leur densité. Nous avons donc admis que le taux de sédimentation varie en fonction de $Q_{N}$ ou de $Q_{P}$ selon une " loi de minimim " pondérée par la durée de la période de carence.

(5) Les apports de $\mathrm{N}$ et de $\mathrm{P}$ en provenance du bassin versant sont estimés à partir d'un recensement des principaux points de rejet (données Agence de Bassin Adour-Garonne) en considérant que un équivalent habitant $=3 \mathrm{~g}$ de $\mathrm{P}$ et $13 \mathrm{~g}$ de $\mathrm{N}$ par jour, dont $50 \%$ sous forme $\mathrm{N}-\mathrm{NO}_{3}$ et $\mathrm{P} \mathrm{PO}_{4}$. Les apports diffus et les échanges à l'interface sédiment-eau ne sont, par contre, pas considérés.

(6) La minéralisation de l'azote organique et la libération de $\mathrm{PO}_{4}$ à partir du $\mathrm{P}$ particulaire et organique dissous sont décrites par des cinétiques de premier ordre dont les taux de réaction $\left(\mathrm{d}_{\mathrm{N}}\right.$ et $\left.\mathrm{d}_{\mathrm{P}}\right)$ ont été évalués par calibration.

La résolution numérique de ce système d'équation consiste à calculer par itérations successives, à 
Tableau I. - Equations utilisées dans le modèle de production de phytoplancton.

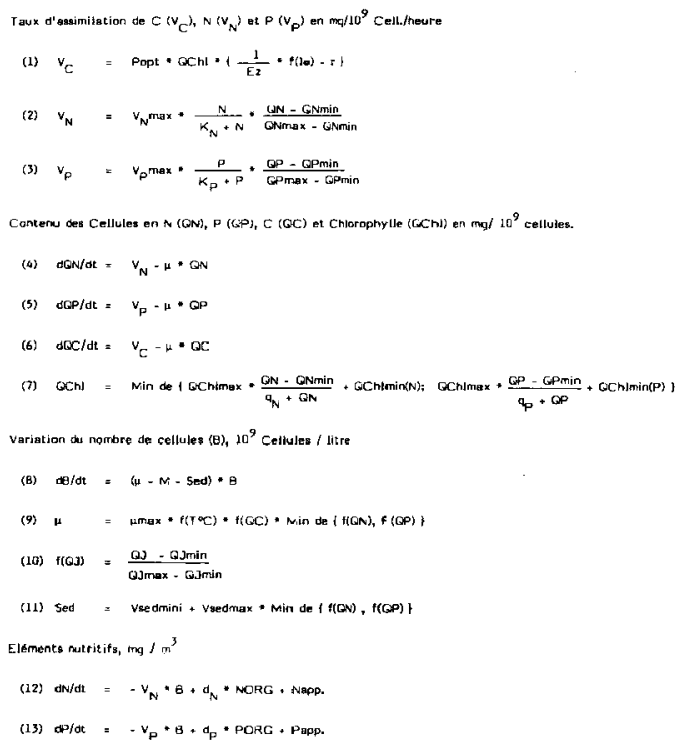

partir d'un état initial donné et compte-tenu des variables de forçage (énergie solaire, débits, apports exogènes) l'évolution amont-aval des variables d'état : biomasse de phytoplancton ; teneurs en Chlorovhylle, carbone, azote, phosphore; quantités de $\mathrm{NO}_{3} . \mathrm{N}$ et de $\mathrm{PO}_{4}$. $\mathrm{P}$ dissous. Ces calculs sont réalisés avec un pas de temps de 1 heure en affectant aux paramètres les valeurs figurant dans le tableau II.

\section{Les données expérimentales}

Les données utilisées pour calibrer et valider ce modèle proviennent de deux séries de mesures effectuées chacune sur une mème masse d'eau pendant son écoulement vers l'aval (fig. 1).

La première série de mesures a été réalisée au mois d'août sur un parcours de $52 \mathrm{~km}$ pour un débit pseudo-régularisé à $15-18 \mathrm{~m}^{3} . \mathrm{s}^{-1}$. Un marquage à la rhodamine a permis de repérer le déplacement de l'eau.
La seconde série, réalisée en juillet, couvre un secteur compris entre le confluent Lot-Truyère $(\mathrm{km} \mathrm{O})$ et Albas ( $\mathrm{km}$ 193). Elle correspond à une période d'étiage sévère pendant laquelle le débit moyen était inférieur à $10 \mathrm{~m}^{3} . \mathrm{s}^{-1}$. La localisation des stations de mesure était déterminée quotidiennement à l'aide d'un modèle simple qui permettait de calculer la vitesse moyenne d'écoulement en fonction des débits et de la morphométrie.

Les relevés ont porté sur la mesure quotidienne des variables suivantes:

Débits moyens horaires : stations de mesure d'Entraygues $(\mathrm{km} 0)$ et de Cajarc $(\mathrm{km} \mathrm{100})$;

Rayonnement solaire incident : pile solarimétrique + enregistreur Kipp et Zonen ;

Transmission de la lumière dans l'eau : quantamère LI-COR avec sonde immergeable :

Eléments minéraux dissous : $\mathrm{CO}_{2}(\mathrm{pH}$ et alcalinité ; $\mathrm{NO}_{3}$ - N (méthode décrite par Rodier, 1978) ; $\mathrm{PO}_{4}-\mathrm{P}$ (méthode décrite par Stephens 1963) : 
Tahleat II. - Valeurs des parameres utilines dans le modeles.

\begin{tabular}{|c|c|c|c|}
\hline (1) & E & Coefficient dextinction do la lumibre & $1,15+0,015 * \mathrm{Cnl}(\mathrm{mg} / \mathrm{ms})$ \\
\hline (2) & Popt & Phatoeynthtere en jumitre sakurente & $3,5.7 \mathrm{mg} \mathrm{C} / \mathrm{mg} \mathrm{Ch}: / \mathrm{h}$ \\
\hline$(3)$ & tk & Intenasite souil de saturation & $180+275 \mu \mathrm{E} / \mathrm{m} 2 / \mathrm{s}$ \\
\hline (4) & umax & Taux de croinance optimal & $1,5 \cdot 25 \subset$ \\
\hline (s) & KN & Const unite de demi-saturation pour $\mathrm{NOS}-\mathrm{N}$ & $2 B, D \mathrm{mg} / \mathrm{ms}$ \\
\hline (6) & kP & Constante de demi-sakuration pour POA-P & $12,5 \mathrm{mg} / \mathrm{ms}$ \\
\hline$\{n$ & Vhimex & Viterme maximale $d^{\prime}$ absorbtion de NO3-N & $675 \mu \mathrm{\mu g} / 10^{9}+/ \mathrm{h}$ \\
\hline (8) & VPmax & Vitose maximale d'abnortion de PO4-P & $375 \mathrm{\mu g} / 10^{9} t / \mathrm{h}$ \\
\hline$(9)$ & ONmax & Contenu meximum en $\mathbf{N}$ des cellules & $4,50 \mathrm{mg} / 10^{9}$ \\
\hline (10) & CPmax & Contern maximum on P des cellultes & $1,25 \mathrm{mg} / 10^{9}$ \\
\hline (11) & Grmin & Conternu minimum en $\mathrm{N}$ des cellulea & $1,50 \mathrm{mg} / 10^{9} \%$ \\
\hline$(12)$ & OPmin & Contern minimum en $P$ des cellules & $0,15 \mathrm{mg} / 10^{9}$ \\
\hline$(13)$ & OCmax & Contern maximum an C dea cellules & $62,5 \mathrm{mg} / 20^{9}$ \\
\hline (14) & @,min & Contenu minimum en $C$ des cellules & $12,5 \mathrm{mg} / 10^{9} \%$ \\
\hline$(15)$ & DC.HLm & Conteme maximum des cellules en Chlorophylle & $650 \% 9 / 10^{9}$ \\
\hline (16) & QCHLminu $(N)$ & teneur minimelo en chlorophylle (cerence en $N$ ) & $100 \mu 9 / 10^{9}$ \\
\hline (17) & $\operatorname{QCH} \min (P)$ & Terneur minimale en chlorophylle (carence en P) & $450 \mathrm{\mu g} / 10^{\circ} \mathrm{F}$ \\
\hline (LB) & VSEDmini & I aux de sedimentation minimum & $0,05 \mathrm{~m} / \mathrm{i}$ \\
\hline (19) & VSEDMaxi & Taux de sedimentation maximum & $0,10 \mathrm{~m} / \mathrm{j}$ \\
\hline$(20)$ & $\mathbf{d}$ & Toux de requntration de $\mathrm{MOB}-\mathrm{N}$ & $0,0<5 / j$ \\
\hline (21) & $\mathbf{P}$ & Toux de requnaration de POAP & $0,10 / \mathrm{i}$ \\
\hline (22) & $\mathbf{r}$ & Rapport Reapiration / Popt & 0,1 \\
\hline
\end{tabular}

- Chlorophylle : dosages spectrophotométriques sur extraits acétoniques (série $\mathrm{n}^{\circ} 1$ ) ou alcooliques (série $\mathrm{n}^{\circ} 2$ ) combinés avec des mesures de fluores. rence * in vivo " (série $n^{\circ} 2$ ) pour détecter d'éventuelles hétérogénéités dans le secteur de nrélèvements ;

- Biomase de phytoplancton : comptage des cellules, détermination du biovolume moyen des espèces présentes et calcul du Carbone cellulaire à l'aide des relations établiées par Strathman (1967) (série $\mathrm{n}^{\circ} 1$ ); mesure des quantités de carbone. d'azote et de phosphore des matières en suspension (série $n^{\circ} 2$ ): la corrélation obtenue entre le $\mathrm{C}$ et le $\mathrm{N}$ organique particulaire (combustion + dosage par Chromatographie du $\mathrm{CO}_{2}$ et $\mathrm{N}_{2}$ - Dalper 1982) et la Chlorophylie $(r \geqslant 0,85)$ indiquent oue ces deux éléments proviennent, pour l'essentiel, du phytoplancton ;

Activité photosynthétique des algues: mesures nuotidiennes sur des profils verticaux par la méthode $\mathrm{du}{ }^{14} \mathrm{C}$. Les mesures réalisées au laboratoire sur des cultures de Chlorophycées ont permis par ailleurs de fixer les valeurs de la plupart des paramètres du modèle figurant au tableau II : taux d'absorption des éléments nutritifs, constantes de semi-saturation, contenu minimum et maximum des algues en $\mathrm{C}, \mathrm{N}, \mathrm{P}$ et Chlorophylle.

\section{Résultats}

Les figures 3 à 6 reproduisent l'évolution des variables d'état mesurées sur le parcours CajarcCahors ainsi que les courbes de simulation fournies par le modèle. Les courbes simulées en affectant différentes valeurs au taux de respiration-r. (fig. 3), à la composition initiale des algues- $Q_{c}, Q_{N}, Q_{P} \cdot(f i g$. 4), aux constantes de régénération des nitrates $\left(d_{N}\right)$ et des phosphates $\left(d_{\mathrm{p}}\right)($ fig. 5$)$ et aux apports exogènes de nutriments (fig. 6) permettent d'apprécier l'importance de ces facteurs sur le comportement 

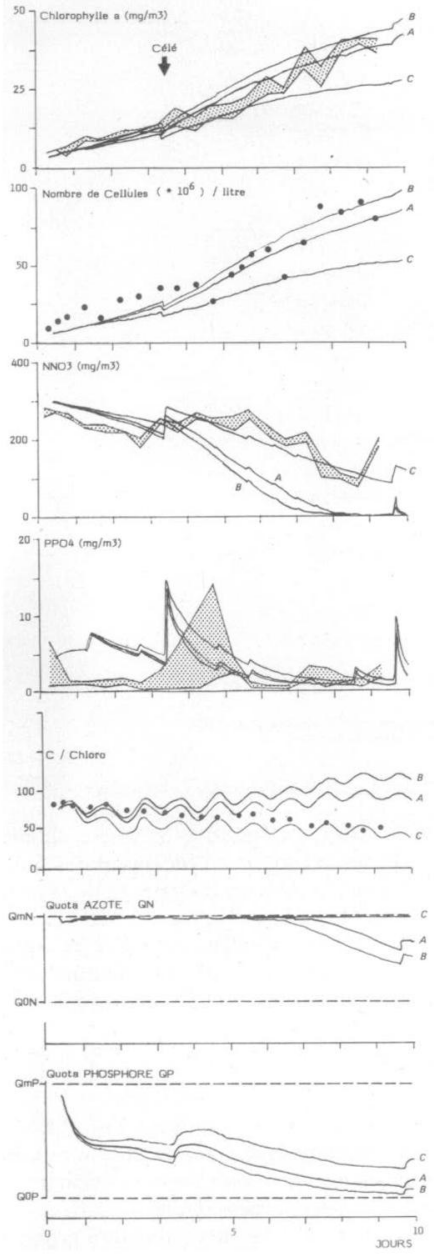

Fig. 3 : Evolution des variables d'etat mesurées tpoints et enveloppes des valeurs en grisé) dans une même masse d'eau sur un parcours de $52 \mathrm{~km}$ (durée $=10$ jours). Les simulations (courbes) correspondent à des rapports Resp/P opt de 0,1 (A), 0,05 (B), et 0,2 (C).
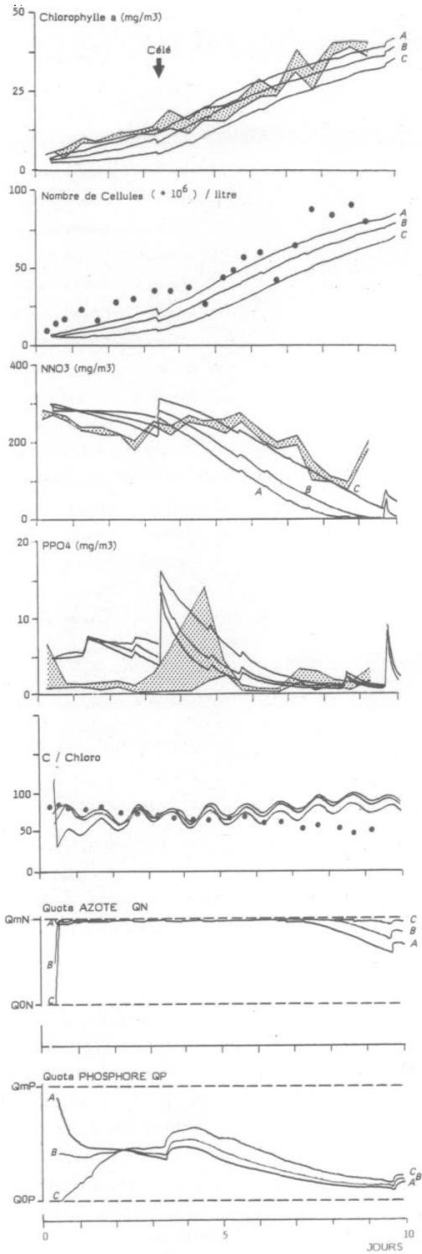

Fig. 4 : Résultats de simulations montrant l'influence de la composition initiale des algues. $Q_{\mathrm{C}}: \mathrm{Q}_{\mathrm{N}}: \mathrm{Q}_{\mathrm{P}}\left(\mathrm{mg} .10^{9}\right.$ cell. -1$)=15: 4,5: 1,25$ (A) $; 30: 2,25: 0,65$ (B) ; $60: 1,5: 0,15$ (C). 

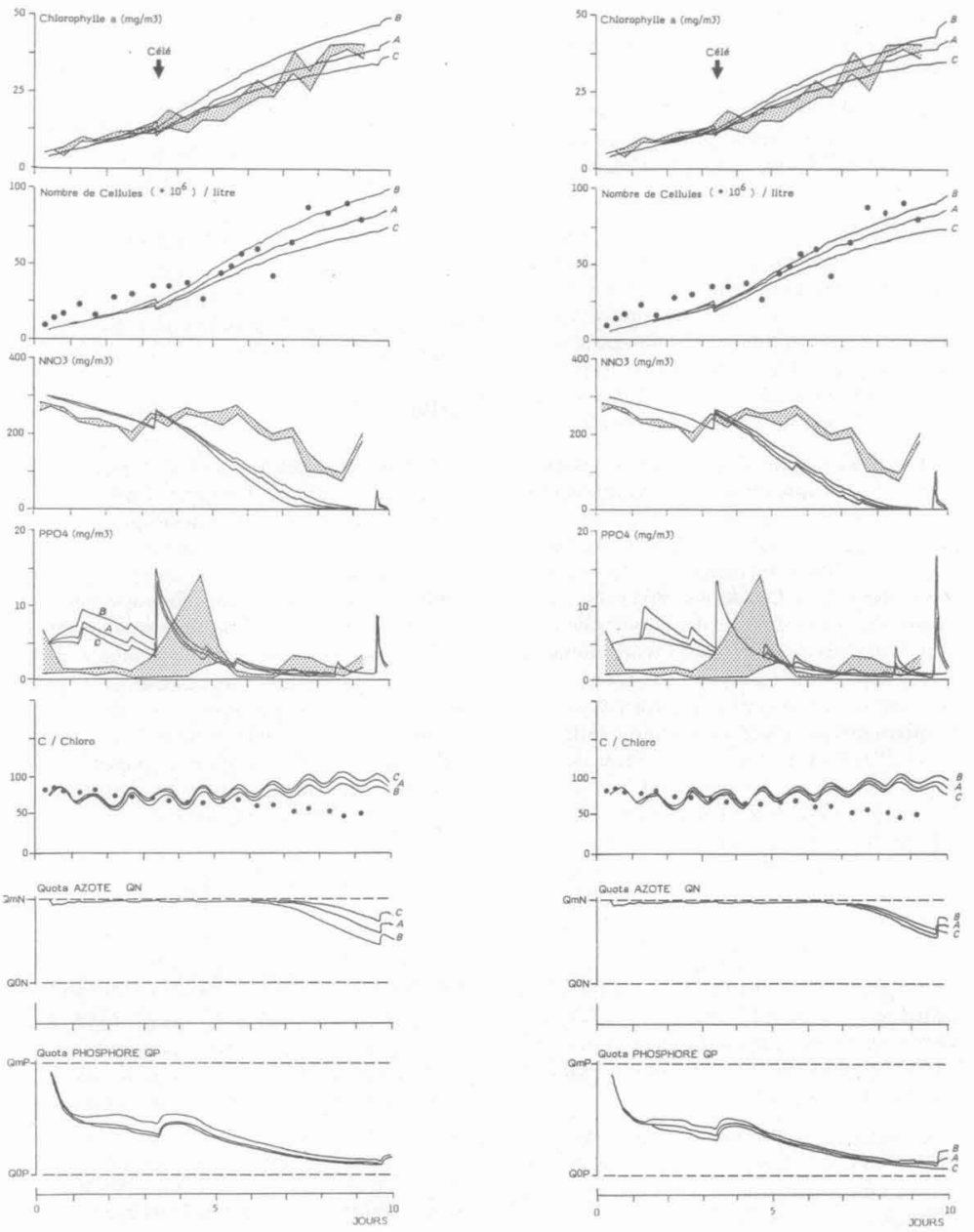

Fip. 5 : Résultats de simulations montrant l'influence des taux de régénération de $\mathrm{NO}_{3}\left(\mathrm{~d}_{\mathrm{N}}\right)$ et de $\mathrm{PO}_{4}(\mathrm{~d}$ ) à partir du matériel détritique. $A: d_{n}=0,045 j^{-1}$ et $d_{P}=0,1$ $i^{-1} ; B: d_{N}=0,1 j^{-1}$ et $d_{P}=0,2 j^{-1} ; C: d_{N}=0,02$ $i^{-1}$ et $d_{p}=0,05 j^{-1}$.

Fig. 6: Resultats de simulations obtenus lorsque les apports de $\mathrm{N}$ et $\mathrm{P}$ estimés par Eq. hab. (A) sont doublés (B) ou supprimés (C). Les apports du Célé (mesurés) restent constants dans les trois cas. 
du modèle. Dans ces différentes simulations, les aut res paramètres conservent les valeurs données au tableau II.

Les tendances observées sur ce secteur de rivière sont relativement bien reproduites par le modèle, les tests de sensibilité aux paramètres révélant en particulier les points suivants.

1) La turbidité des eaux de la rivière exerce un contrôle sur le développement des algues planctoniques par le biais du bilan photosynthétique. Ce mécanisme, déjà mis en évidence dans les études précédentes (Capblancq \& Décamps 1978, Décamps \& al 1984, Capblancq \& al 1982) apparait ici lorsqu'on considère les conséquences des modifications du raoport $\mathbf{r}$ (R/Popt) sur les sorties du modèle.

2) Les quantités de $\mathrm{PO}_{4}-\mathbf{P}$ disponibles limitent apoaremment le développement du phytoplancton. Le modèle indique que les flux de $\mathrm{PO}_{4}$ en provenance du bassin versant et des sédiments sont beaucoup plus importants à considérer que les quantités présentes dans l'eau. Ces dernières représentent en effet une situation résiduelle, les apports de $\mathrm{PO}_{4}$ -P (en particulier ceux du Célé) étant immédiatement mobilisés par le phytoplancton qui poursuit sa croissance au dépens des réserves internes dans un milieu relativement pauvre en phosphates (moins de 2 ug. $1^{-1}$ de $\mathrm{PO}_{4}-\mathrm{P}$ en général). Le niveau de ces réserves internes $\left(Q_{P}\right)$, qui contrôlent le taux de croissance des populations d'algues, explique la sensihilité du modèle (1) aux quantités de $P$ initialement stockées par les algues (2) aux quantités de phosphore rejetées dans la rivière (3) aux cinétiques de libération du $\mathrm{PO}_{4}$ par la fonction détritique ( $\mathrm{P}$ organique dissous et $\mathbf{P}$ des particules).

On ne constate par contre aucun effet semblable pour l'azote, le contenu des cellules en $\mathbf{N}\left(\mathbf{Q}_{\mathrm{N}}\right)$ se maintenant à un niveau proche du maximum quel que soient les quantités importées, l'état initial des aloues ou les taux de minéralisation.

La validation de ce modèle est assurée par la comparaison des courbes simulées avec les données mesurées sur un parcours de près de $200 \mathrm{kms}$ ( $f \mathrm{ig}$. 7). L'évolution des variables d'état (biomasse de phytoplancton, concentration en éléments nutritifs, composition élémentaire des algues) est assez fidèlement reproduite par le modèle. L'épuisement des ressources nutritives se traduit en particulier par une modification des rapports entre constituants de la biomasse qui indiquent un état de carence dans la partie centrale du parcours simulé. Ainsi, les rapports $\mathrm{C} / \mathrm{CHL}, \mathrm{P} / \mathrm{CHL}, \mathrm{C} / \mathrm{N}, \mathrm{N} / \mathrm{P}$ varient dans des limites tout à fait identiques à celles que Healey (1978), Healey \& Hendzel (1979) ont définies sur des cultures d'algues et des peuplements naturels.

Les techniques de mesure du carbone, de l'azote et du phosphore (qui englobent les constitutants du phytoplancton et du matériel détritique) et la structure du modèle (qui implique en particulier un rapport R/Popt constant) expliquent sans doute des divergences qui apparaissent avec la simulation des rapports $\mathrm{P} / \mathrm{CHL}$ et $\mathrm{C} / \mathrm{N}$.

\section{Discussion}

Le Lot représente un exemple type de rivière aménagée par l'homme. L'intérêt des études réalisées sur ce milieu réside dans la simplification relative du système (absence de stratification verticale, très faible développement de zooplancton) et dans la possibilité de suivre l'évolution des variables d'état dans une mème masse d'eau pendant son écoulement vers l'aval. Les résultats obtenus démontrent qu'il est possible de simuler l'ensemble des interactions physiques-chimiques-biologiques dont cette masse d'eau est le siège pour prévoir l'évolution du peuplement phytoplanctonique à partir de quelques variables externes (débit, ensoleillement, quantités d'éléments nutritifs importés).

Le modèle numérique utilisé pour cette simulation reprend dans sa formulation, la plupart de nos connaissances actuelles sur l'écophysiologie des algues planctoniques. Le comportement de ce modèle confirme les observations effectuées sur le terrain, en particulier sur les points suivants:

1) Les biefs relativement peu profonds de l'amont sont les secteurs de rivière les plus favorables à la croissance du phytoplancton pour des débits inférieurs à $40 \mathrm{~m}^{3} . \mathrm{s}^{-1}$. Vers l'aval, l'augmentation de la profondeur de la rivière alliée à la turbidité de l'eau diminue lá durée d'exposition des algues à la lumière d'où une baisse de la production nette et une stabilisation ou un déclin de la biomasse.

2) La multiplication des algues peut se poursuivre dans une eau très appauvrie en phosphate (souvent moins de $1 \mu \mathrm{g} . \mathrm{I}^{-1}$ de $\mathrm{PO}^{4}$-P en été) grâce à l'aptitude des cellules à stocker cet élément lorsqu'il est fourni en excès. Cette croissance continue vers l'aval 

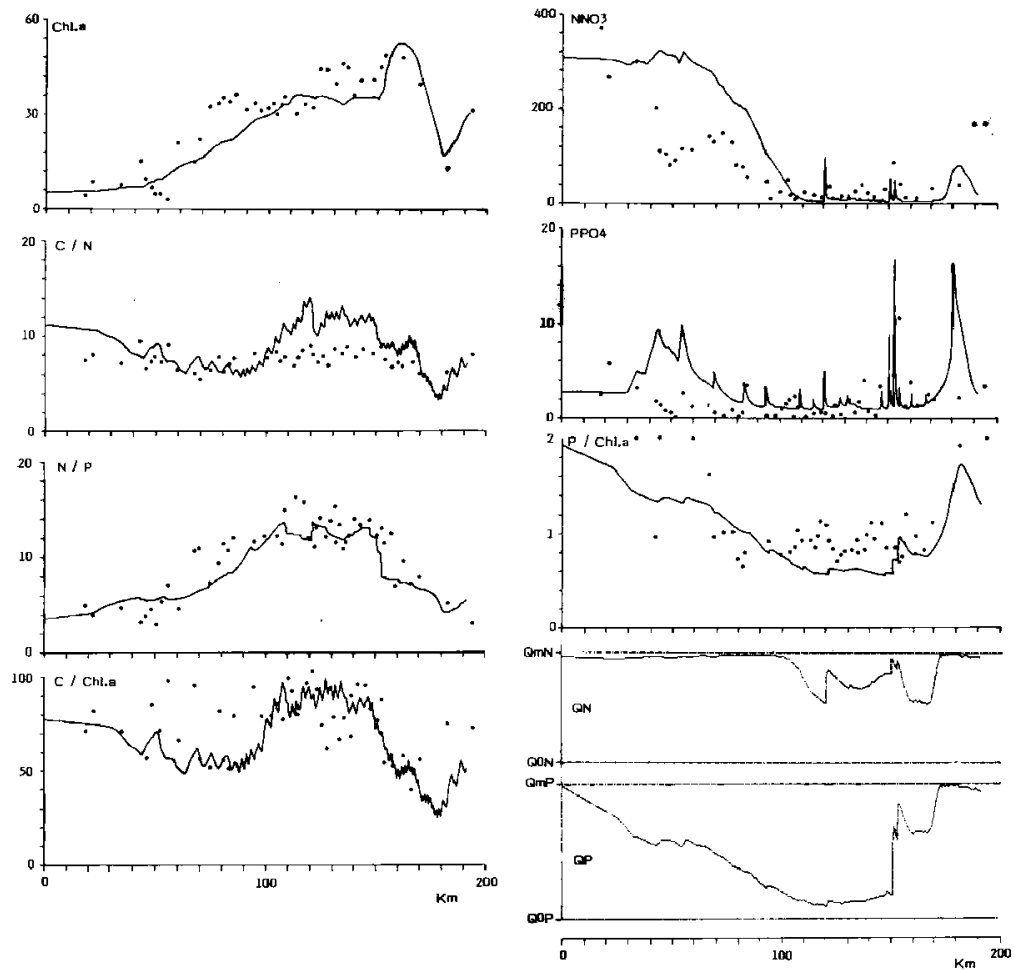

Fï, 7 : Evolution des constituants de la biomasse de phytoplancton chloruphylle, rapports CiN,N/P,C/Chlorophylle, p/chlorophylle) des nitrates et des phosphates dans une même masse d'eau sur un trajet de $195 \mathrm{~km}$ : points mesurés et rourbes simulées.

au dépens des réserves accumulées jusqu'à ce que la masse d'eau en écoulement reçoive un nouvel apvort de phosphates que les algues mobiliseront très rapidement.

3) Les modifications de la composition biochimique des cellules qui résultent de ces changements des conditions nutritives agissent en retour sur le bilan en carbone (variations du rapport $P / R$ ) et sur les pertes par sédimentation.
Sur ces points, il apparaît cependant nécessaire d'affiner la structure du modèle en considérant notamment : - les changements de populations dominantes d'algues fréquemment observés entre l'amont et l'aval de la rivière (Dauta 1975) et les variations des paramètres liés à ces changements ;

- les variations des pertes par respiration en fonction de la taille des cellules (Banse 1976), de leur contenu en hydrates de carbone (Gibson 1978) et de leur état nutritionnel ; 
- les quantités de phosphore biodisponible effectivernent rejetées dans la rivic̀rc et les cinétiques des échanges qui s'établissent entre le $P$ des microorgan ismes, des sédiments en suspension et des sédiments déposés.

Le risque est évidemment de déboucher sur un modèle plus complexe avec un tel nombre de paramètres à identifier que le gain en précision ne serait peut-être qu'illusoire.

Dans sa formulation actuelle et malgré les simplifications apportées dans la représentation des mécanismes, ce modèle parait cependant pouvoir être utilisé pour apprécier les effets des divers schémas d'aménagement envisagés : renforcement des débits d'étiage ou réduction des rejets de nutriments dans la rivière.

\section{Remerciements}

Nous remercions J.N. Tourenq, Chargé de recherches au C.N.R.S, pour l'aide efficace apportée dans la collecte des données de terrain, C. Mur et D. Dalger qui ont effectué les analyses chimiques.

\section{Travaux cltés}

Banse (K.). 1976. - Rates of growth, respiration and photosynthesis of unicellular algae as related to cell size. A review. $J$. Phycol., 12 : 135-140.

Brinel (L.), Dauta (A.) \& Guerri (M.M.). 1982. - Croissance algale : validation d'un modèle à stock à l'aide de données expérimentales. Annls. Limnol, 18 : $91-99$.

Canblancq (J.) \& Dauta (A.). 1978. - Phytoplancton et production primaire de la rivière Lot. Artnls. Limnol, $14: 85-112$.

Canblancq (J.) \& Décamps (H.). 1978. - Dynamics of the phyto plankton in the river lot. Verh. Int. Ver. Theor. Angew. Limnol., 20 : $1479-1484$

Canblancq (J.), Dauta (A.), Décamps (H.) \& Caussade (B.). 1982. Variations journalières de la production du phytoplancton en rivière: modélisation d'un bief du Lot. Annls. Limmol., 18 : 101-132.

Cacanova.Batut (T.), 1977. - Etude préliminaire en vue de la modélisation de l'écosystème Lot. These $3^{\circ}$ cycle, Toulouse., 109 p.
Caussade (B.), Chaussavoine (C.), Dalmayrac (S.) \& Masbernat (L.). 1978. - Modélisation d'écosystèmes rivières : application à un bicf du Lot. Annls. Limnol., $14: 139.162$.

Dalger (D.). 1982. - Dosage du carbone et de l'azote organiques dans l'eau par chromatographie en phase gazeuse: mise au point et application de la méthode. Thèse 3 eycle, Toulouse: $75 \mathrm{P}$

Dauta (A.). 1982. - Conditions de développenent du phytoplancton : étude comparative du comportement de huit especes en cultures. II. Róle des nutriments; assimilation et stockage. Annls. Limnol, 19 : 73-86.

Dauta (A.), 1983. - Conditions de développement du phytoplancton. Etude comparatjve du comportement de huit especes en culture, cinetiques d'assimilation et de croissance : étude expos rimentale, modélisation appliquée aux cultures et à un milieu naturel (Le Lot). These Doxtorat, Univ. Toulouse: $166 \mathrm{p}$.

Dauta (A.), Brunel (L.) \& Guerri (M.M.). 1982. - Determination experimentale des paramètres liés à l'assimilation de l'azote et du phosphore par Scenedesmus crassus. Annls. Limnol., 18 : 33-40.

Décamps (H.), Capblancq (J.) \& Tourenq (J.N.). 1984. - Lot. in Whitton, B.A. (ed.), Ecology of European rivers, Blackwell Sciertific Publications : 207-235.

Delclaux (F.). 1980. - Production primaire en milieu thermiquement stratifié. Modélisation et application à la rivière Lot. Thèse Docteur Ingénieur, INP Toulouse, $177 \mathrm{p}$.

Guerri (M.M.), Brunel (L.) \& Dauta (A.). 1981. - Interaction de la lumière et de la température sur le taux de croissance de Scenedesmus crassus. Annls. Limmol, 17:97-104.

Gibson (C.E.). 1978. - Field and laboratory observations on the temporal and spatial variation of carbohydrate content in planktonic blue - green algae in Lough Neagh, Northern Ireland. J. Ecol., $66: 97.115$

Healey (F.P.). 1978. - Physiological indicators of nut rient deficiency in algae. Mitt. Int. Ver. Theor. Angew. Limnol, $21: 34-41$.

Healey (F.P.) \& Hendzel (L.L.). 1979. - Indicators of phosphorus and nitrogen deficiency in five algae in culture. $J$. Fish. Res. Board Can., $36: 1364-1369$.

Lehman (J.T.). Botkin (D.B.) \& Likens (G.E.). 1975. - The assumptions and rationales of a computer model of phytoplankton population dynamics. Limnol. Oceanogr., 20 : 343-364.

Nyhoim (N.). 1977 a. - Kinetics of phosphate limited algal growth. Biolechnol. Bioengineer., $9: 467-492$.

Nyholm (N.). 1977 b. - Kinetics of nitrogen limited algal growth Prog. wot. Tech., $8: 347$ - 358.

Rhee (G.Y.). 1978. - Effets of N : P atomic ratios and nitrate limitation on algal growth, cell composition and nitrate uptake. Limnol. Oceanogr., $23: 10-25$.

Rodier (J.). 1978. - Lanalyse de l'eau. Dunod, 6c edition, $1136 \mathrm{p}$.

Stephens (K). 1963. - Determination of low phosphate concentration in lake and marine waters. Limnol Oceanogr. 8: $361-362$

Strathman (R.R.). 1967. - Estimating the organic carbon content of phytoplankton from cell volume or plasma volume. LimnoL. Oceanogr., $12: 411-418$. 